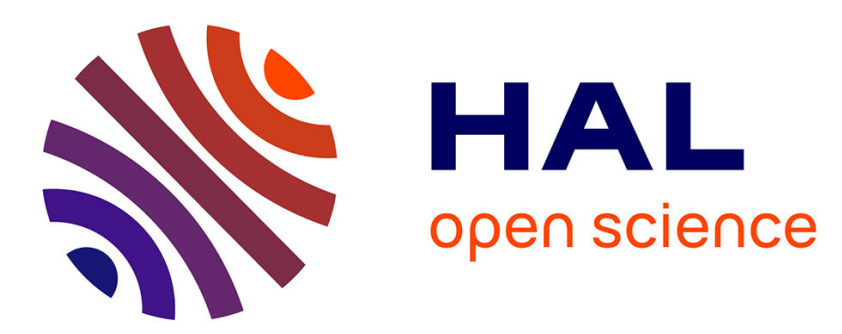

\title{
An easy-to-use method for measuring the flux of free atoms in a cluster beam
}

J. Cuvellier, A. Binet

\section{To cite this version:}

J. Cuvellier, A. Binet. An easy-to-use method for measuring the flux of free atoms in a cluster beam. Revue de Physique Appliquée, 1988, 23 (1), pp.91-96. 10.1051/rphysap:0198800230109100 . jpa-00245751

\section{HAL Id: jpa-00245751 https://hal.science/jpa-00245751}

Submitted on 1 Jan 1988

HAL is a multi-disciplinary open access archive for the deposit and dissemination of scientific research documents, whether they are published or not. The documents may come from teaching and research institutions in France or abroad, or from public or private research centers.
L'archive ouverte pluridisciplinaire HAL, est destinée au dépôt et à la diffusion de documents scientifiques de niveau recherche, publiés ou non, émanant des établissements d'enseignement et de recherche français ou étrangers, des laboratoires publics ou privés. 


\title{
An easy-to-use method for measuring the flux of free atoms in a cluster beam
}

\author{
J. Cuvellier and A. Binet \\ Service de Physique des Atomes et des Surfaces, Centre d'Etudes Nucléaires de Saclay, 91191 Gif-sur-Yvette \\ Cedex, France
}

(Reçu le 21 juillet 1987, accepté le 12 octobre 1987)

\begin{abstract}
Résumé. - On décrit une méthode originale permettant de déterminer, dans un faisceau atomique produit par détente supersonique et composé en majorité d'agrégats, la quantité d'atomes restés à l'état de monomères. Pour le cas de l'argon considéré ici, les spectres de vitesse des monomères et des agrégats, mesurés à la masse 40 , présentent une structure bimodale qui évolue avec la concentration et la taille des agrégats. La composante lente est associée aux agrégats qui se sont fragmentés dans la chambre d'ionisation. Le mode rapide est attribué aux monomères, et l'intégrale de ce mode est proportionnelle au flux de ces monomères. Des observations identiques ont été faites dans le cas d'un faisceau de $\mathrm{N}_{2} \mathrm{O}$.
\end{abstract}

\begin{abstract}
A method is proposed to measure the flux of free atoms remaining in a beam of clusters. The timeof-flight (TOF) of an Ar beam containing clusters was analysed for this purpose using an electron impact + quadrupole mass spectrometer as detector. When considering TOF's with mass settings at $\mathrm{Ar}^{+}$, a double mode structure was observed. The slow component was interpreted as coming from Ar clusters that fragment as $\mathrm{Ar}^{+}$in the ionization chamber of the detector. The rapid mode in the TOF's was linked to the free atoms remaining in the Ar beam. Evaluating the area of this mode allowed one to measure the flux of free atoms in the Ar beam. The method is not restricted to measurements on Ar beams.
\end{abstract}

\section{Introduction.}

Beams containing neutral Van der Waals clusters are commonly generated by supersonic expansions. The cluster size distribution is an important parameter which characterizes the beam. It is often measured using an electron impact mass spectrometer. However, fragmentation follows the cluster ionization step. This scrambles the informations that can be derived from the observation of mass spectra. Other ionization techniques such as penning- or photoionization lead to the same difficulties. A number of references concerning fragmentation resulting in cluster ionization are given in reference [1].

The fragmentation processes following ionization are fairly well known for Ar clusters of small size, $(\mathrm{Ar})_{2},(\mathrm{Ar})_{3}$ up to $(\mathrm{Ar})_{6}$ [1]. However, even if available for larger clusters, such an information would be very difficult to use to fully characterize cluster beams. The number of fragmentation channels indeed, increases dramatically with the cluster size.

The analysis of electron diffraction pattern has REVUE DE PHYSIQUE APPLIQUÉE. - T. 23, N-1, JANVIER 1988 allowed to determine the fraction of free Ar atoms contained in a beam of fairly large Ar clusters [2]. This is a first useful step in characterizing cluster beams. This method is however very complex and indirect, and providing such information is not its primary goal. The aim of the present work is to propose an alternate method which is both more direct and easy to use. More precisely, the method presented here allows measurement of the flux of free atoms remaining in a beam containing clusters. It takes advantage of the fact that will be evidenced here that large clusters in an Ar beam do not have exactly the same velocity than remaining free atoms.

The present method is not restricted to argon. For instance, it has been recently used to highlight the changes in reactivity of monomers or clusters of $\mathrm{N}_{2} \mathrm{O}$ with barium atoms [3].

The principle of the method is explained in section 2 of the present work. The experimental apparatus is described in section 3 . The experimental results showing the velocity shift between monomers and large clusters are shown and discussed in section 4. Finally, the flux of free $\mathrm{Ar}$ atoms contained in 
the beam is estimated in section 5 for various conditions of supersonic expansion.

\section{Measuring the flux of free atoms in a cluster beam : principle.}

The formation of clusters in supersonic beam expansions has been studied for a long time [4-5]. Essential features needed for understanding the present measurement method are recalled briefly.

The supersonic expansion of a gas into vacuum results in partial conversion of random energy into kinetic energy directed along the beam. This makes the gas temperature and pressure going down from the stagnation conditions $T_{0}, P_{0}$ to conditions where the gas is possibly supersaturated and condenses. Clusters then form, and their size depends on the stagnation conditions. Larger sizes are associated to lower $T_{0}$ or higher $P_{0}$. Cluster formation during the expansion is associated to an additional energy release because of the cluster binding energies. This results in enhancement of the monomer and cluster velocities. The larger the clusters, the larger this effect [6].

When a supersonic beam is generated from the expansion of a binary light + heavy gas mixture, without forming clusters, the light particles have a larger velocity along the beam axis than the heavy one. The smaller the stagnation pressure $P_{0}$, the larger this velocity shift [7].

Let us go back to cluster beams and consider a supersonic expansion of neat Ar gas under conditions where clusters are formed. The cluster formation region is at distances of several nozzle diameters downstream the nozzle, and the supersonic expansion keep going after clusters are formed. During the late expansion stage, the beam can be considered as operating under Ar cluster/free Ar atom seeding conditions with pressures much smaller than $P_{0}$. By analogy with what seen above for supersonic expansions of gas mixtures, it can be thought that a velocity shift between the free atom component and the cluster component of the Ar beam will result in this late expansion. Evidence of this is given in section 4.

The principle for measuring the flux of free atoms in a cluster beam is then very simple. Time-OfFlights (TOF) are recorded using an electron impact mass spectrometer as a detector. Because of the fragmentation in the ionization chamber of the mass spectrometer, the TOF's recorded for $\mathrm{Ar}^{+}$do not reflect simply the velocity distribution of the free Ar atoms contained in the beam, but also that of the clusters which are present. In the same way TOF's recorded with the mass spectrometer set to $(\mathrm{Ar})_{n}^{+}$ contains information about $(\mathrm{Ar})_{n}$ and larger clusters. Let us consider a TOF recorded for $\mathrm{Ar}^{+}$. The existence of a velocity shift between the free Ar atoms and the clusters will serve to distinguish between the $\mathrm{Ar}^{+}$ions corresponding to free $\mathrm{Ar}$ atoms, and those corresponding to fragmented ionized clusters. This will allow one to derive quantitative information on the flux of free atoms contained in the Ar beam.

\section{Experimental apparatus.}

The perturber beam arm of a crossed beam machine described elsewhere [8], has been used for the present work. Briefly, the cluster beam is generated by supersonic expansion of neat $\mathrm{Ar}$ gas at room temperature for stagnation pressures ranging between 0.5 and 15 bars. Nozzles of 0.1 and $0.2 \mathrm{~mm}$ diameter were used.

The beam particles are detected by a quadrupole mass spectrometer with ionization performed by $40 \mathrm{eV}$ electron impact. A time-of-flight system allows measurement of the velocity and velocity distribution of $\mathrm{Ar}^{+}$up to (Ar) $)_{6}^{+}$.

A Pitot tube placed in the beam serves as a gauge giving the total flux of Ar atoms in the beam, wherever they are, free or associated in clusters. The Ar clusters formed in the supersonic expansion are indeed totally dissociated as Ar atoms when thermalized into the Pitot tube chamber.

\section{Experimental results and discussion.}

TOF's recorded for $\mathrm{Ar}^{+},(\mathrm{Ar})_{2}^{+}$and $(\mathrm{Ar})_{3}^{+}$are shown in figures 1-3 for the values $0.3,0.5$ and $1.5 \mathrm{bar} . \mathrm{mm}$ of the product $P_{0} \cdot d\left(P_{0}\right.$ is the stagnation pressure and $d$ the nozzle diameter). Figure 4 gives the TOF's for $\mathrm{Ar}^{+}$up to $(\mathrm{Ar})_{6}^{+}$at a $P_{0} \cdot d$ of 3 bar . $\mathrm{mm}$.

Figures 1 to 4 correspond to larger and larger values of the product $P_{0} \cdot d$, i.e. to situations where the clusters are larger and larger and where they are more and more abundant. Several features must be noticed :

i) Whatever the value of $P_{0} \cdot d$, the TOF's for $(\mathrm{Ar})_{2}^{+},(\mathrm{Ar})_{3}^{+}, \ldots$ are bell shaped.

ii) In contrast, the TOF measured for $\mathrm{Ar}^{+}$has a double structure for certain values of $P_{0} \cdot d$. This is the most prominent observation in figures 1-4.

iii) When two structures are present in the $\mathrm{Ar}^{+}$TOF, the slow mode has the same peak velocity than the TOF's measured for the other mass settings $(\mathrm{Ar})_{2}^{+},(\mathrm{Ar})_{3}^{+}, \ldots$

Of course, since the $\mathrm{Ar}^{+}$signal gives information on both the free Ar atom and Ar cluster TOF's, the double structure seen in the $\mathrm{Ar}^{+}$TOF's is likely attributed to the velocity shift announced in section 2 between the cluster component and the free atom component of the Ar beam. This statement is substantiated now by discussing figures $1-4$ in more details. 
Figure 1 corresponds to a regime where only few clusters are present, and where they are small. It is therefore not surprising that the TOF for $\mathrm{Ar}^{+}$is adequately fitted by the standard expression :

$S(t)=A \times$

$$
\times \exp \left[-m V^{2}\left(t_{0} / t-1\right)^{2} /(2 k T)\right] / t^{4}
$$

giving the TOF distribution resulting in supersonic expansion of neat gases [9]. In (1), $S(t)$ is ion

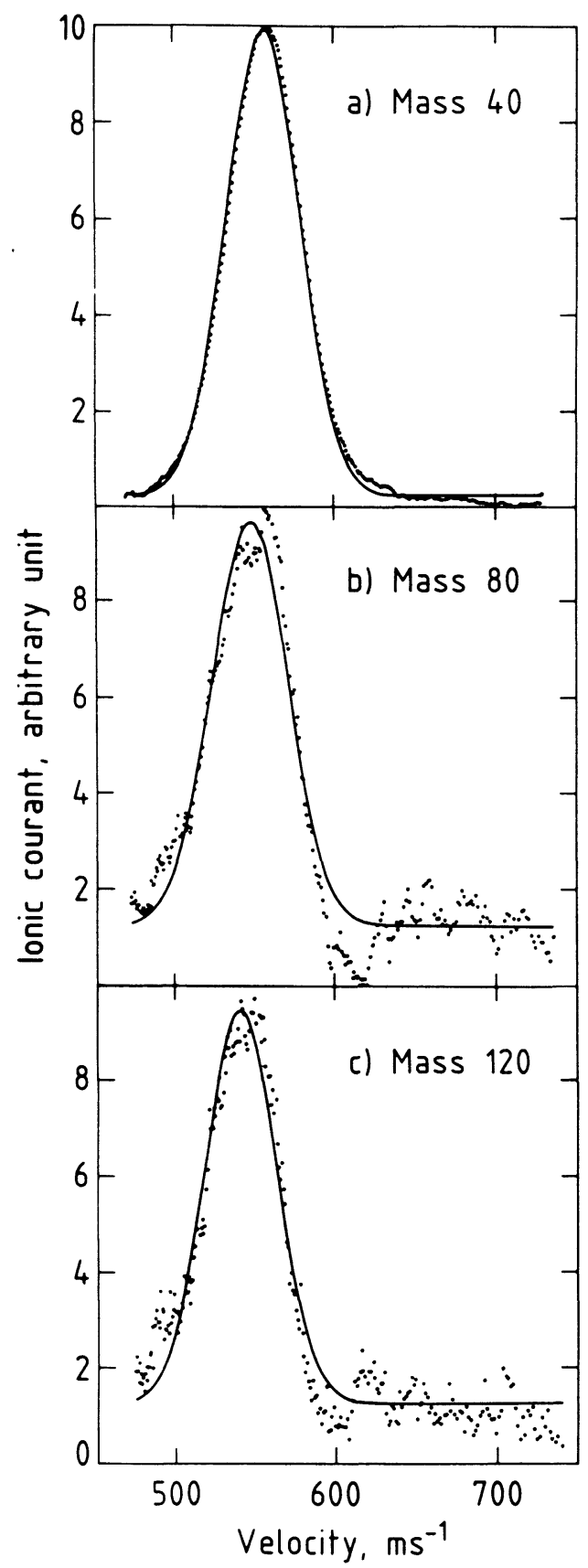

Fig. 1. - Time-of-Flight recorded with the mass spectrometer set to masses of $\mathrm{Ar}^{+}$(a), ( $\left.\mathrm{Ar}\right)_{2}^{+}$(b) and (Ar) $)_{3}^{+}$(c). The product $P_{0}, d$ is 0.3 bar . mm (see text). The dots are the experimental data, and the solid curves are fits to the data using expression (1). intensity as a function of the time $t ; m$ is the mass of Ar, $V$ is the hydrodynamic velocity and $t_{0}$ the corresponding time of flight, $k$ is the Boltzmann constant and $T$ is the translational temperature. It is interesting to notice that the TOF's measured for the mass of $(\mathrm{Ar})_{2}$ and $(\mathrm{Ar})_{3}$ in figure 1 are fitted by expression (1) as well, of course after giving $m$ the value relevant to $(\mathrm{Ar})_{2}$ or $(\mathrm{Ar})_{3}$. The corresponding peak velocities are significantly smaller than that observed for $\mathrm{Ar}^{+}$.

Figure 2 corresponds to a larger value of the product $P_{0} . d$. In this regime, more clusters are present, and their average size is larger than it was under the conditions of figure 1. The essential change with respect to figure 1 is the double structure which appears in the TOF measured at the mass of $\mathrm{Ar}^{+}$. Expression (1) can be used successfully to fit the TOF's measured at the $(\mathrm{Ar})_{2}^{+}$and $(\mathrm{Ar})_{3}^{+}$ masses, as well as both modes of the $\mathrm{Ar}^{+}$TOF. In that case, the peak velocity for the slow mode is found equal to that fitted in the $(\mathrm{Ar})_{2}^{+}$and $(\mathrm{Ar})_{3}^{+}$TOF's. This is a clue to ascertain that the slow mode in the $\mathrm{Ar}^{+}$TOF is linked to cluster TOF better than to free Ar atom TOF.

In figure 3 , the product $P_{0} \cdot d$ and correspondingly the size and the amount of Ar clusters have increased with respect to the conditions of figure 2 . The slow mode in the $\mathrm{Ar}^{+} \mathrm{TOF}$ is now dominant, and corresponds to the same velocity than the peak in $(\mathrm{Ar})_{2}^{+}$and $(\mathrm{Ar})_{3}^{+}$TOF's. It is associated to a substantially larger velocity than the corresponding peak in figure 2 . This substantiate the expectation of section 2 that bond formation in clusters should result in additional energy release in the supersonic expansion, the final velocities in supersonic beams thus being larger when clusters are formed than when not. Expression (1) is still valid to fit the rapid mode in $\mathrm{Ar}^{+} \mathrm{TOF}$, but it can no longer be used to reproduce the slow mode of this velocity spectrum, nor the $(\mathrm{Ar})_{2}^{+}$and $(\mathrm{Ar})_{3}^{+}$TOF's. In these cases indeed, there is no way using expression (1) to fit the low velocity tail of the TOF's. This reflect the fact that expression (1) is giving TOF distributions in supersonic expansions for neat gases in conditions where no or almost no clusters are formed. The interesting point here is that this expression can still be used in conditions where a lot of clusters are present, but for describing the TOF distributions of the free Ar atom component of the beam.

Figure 4 corresponds to a situation where clusters are dominant. In contrast with figure 3 , the rapid mode in the $\mathrm{Ar}^{+}$TOF is no longer visible. The single peak observed in figure $4 \mathrm{Ar}^{+}$TOF is indeed essentially what we called the slow mode in figures 2 and 3 , since it corresponds to the same velocity than the peaks in the $(\mathrm{Ar})_{n}^{+}$TOF's.

This discussion of figures 1-4 substantiate the 


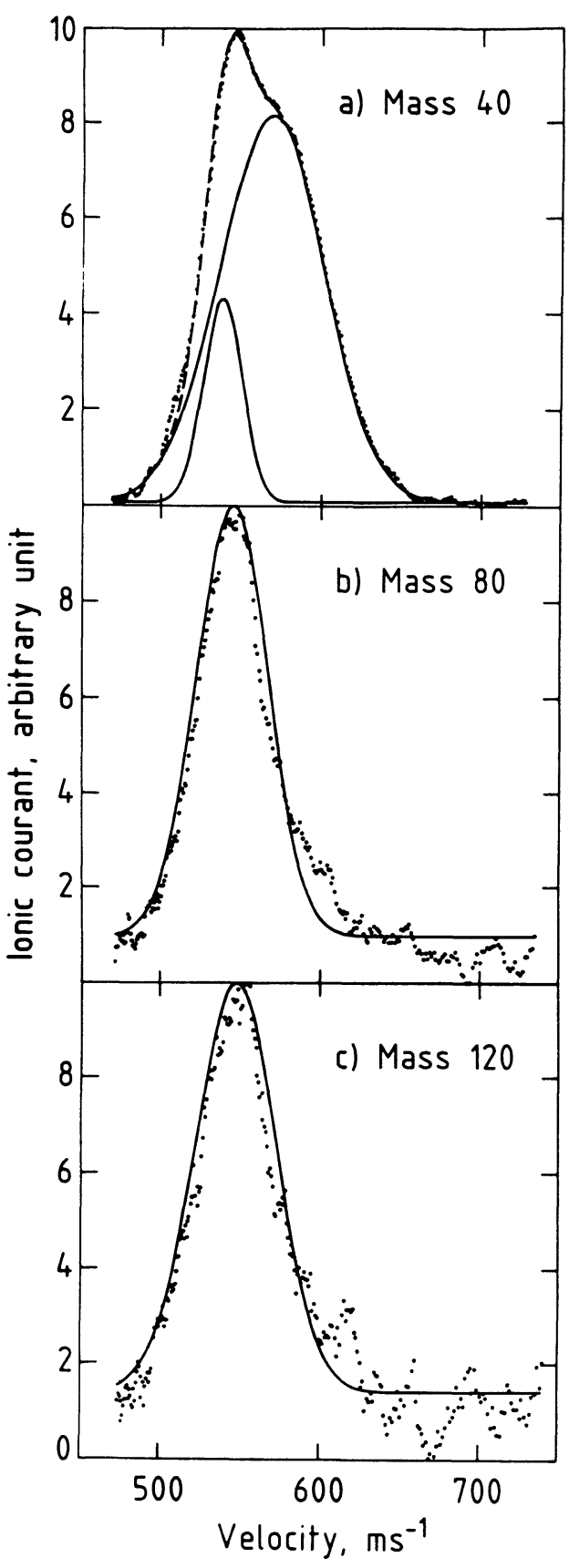

Fig. 2. - Time-of-Flight recorded with the mass spectrometer set to masses of $\mathrm{Ar}^{+}$(a), ( $\left.\mathrm{Ar}\right)_{2}^{+}$(b) and $(\mathrm{Ar})_{3}^{+}$(c). The product $P_{0}, d$ is 0.5 bar . mm (see text). The dots are the experimental data. The dashed curves is a fit to these data. It is the sum of the two modes represented by the solid curves.

statement anticipated above, that clusters fragmented by electron impact show up as a slow mode in the $\mathrm{Ar}^{+} \mathrm{TOF}$. This lets the rapid mode to be associated to the free atom component of the $\mathrm{Ar}$ beam.

The velocity shift between clusters and the free atom component of the Ar beam is betterly seen in figure 5 where the peak velocity corresponding to the rapid peak of the $\mathrm{Ar}^{+}$TOF is plotted as a

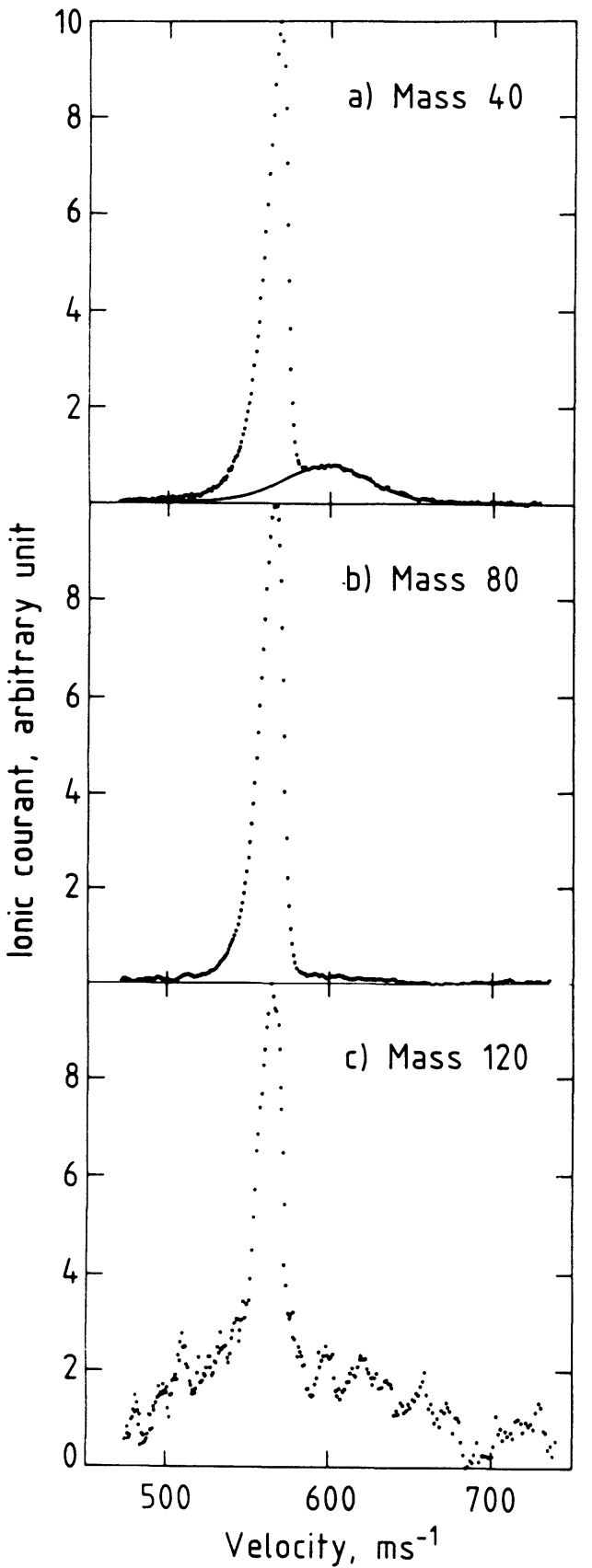

Fig. 3. - Time-of-Flight recorded with the mass spectrometer set to masses of $\mathrm{Ar}^{+}$(a), ( $\left.\mathrm{Ar}\right)_{2}^{+}$(b) and $(\mathrm{Ar})_{3}^{+}$(c). The product $P_{0} \cdot d$ is 1.5 bar . mm (see text). The dots are the experimental data, and the solid curve (a) a fit to the rapid mode.

function of the product $P_{0} \cdot d$, along with the slow peak velocity. The two effects announced in section 2 and already discussed are clearly visible in this figure :

i) A velocity shift exists between the free atoms and the clusters contained in the beam. The larger $P_{0} . d$, the larger this shift.

ii) Velocities of both the free atoms and the clusters are increasing with $P_{0} \cdot d$. This is due to the 


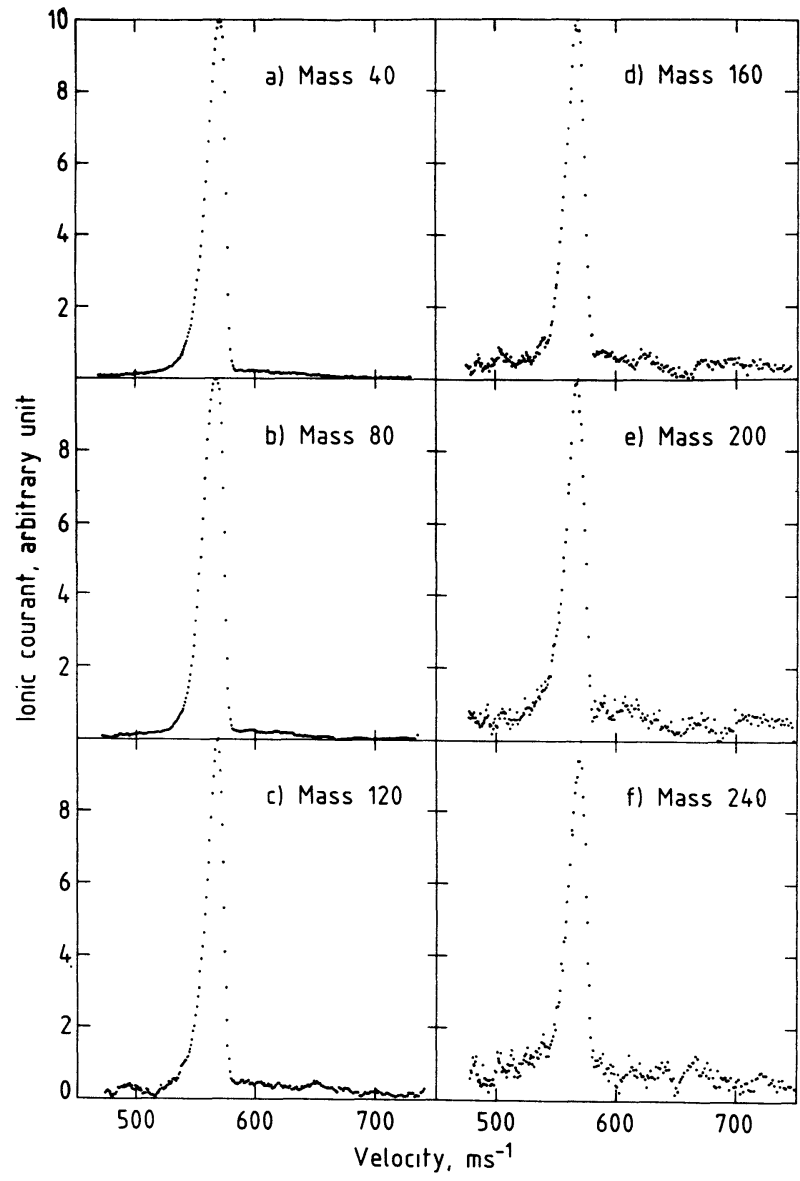

Fig. 4. - Time-of-Flight recorded with the mass spectrometer set to masses of $\mathrm{Ar}^{+}(\mathrm{a}),(\mathrm{Ar})_{2}^{+}(\mathrm{b}),(\mathrm{Ar})_{3}^{+}$(c), $(\mathrm{Ar})_{4}^{+}(\mathrm{d}),(\mathrm{Ar})_{5}^{+} \quad(\mathrm{e})$ and $(\mathrm{Ar})_{6}^{+}$(f). The product $P_{0} . d$ is 3.0 bar. mm (see text).

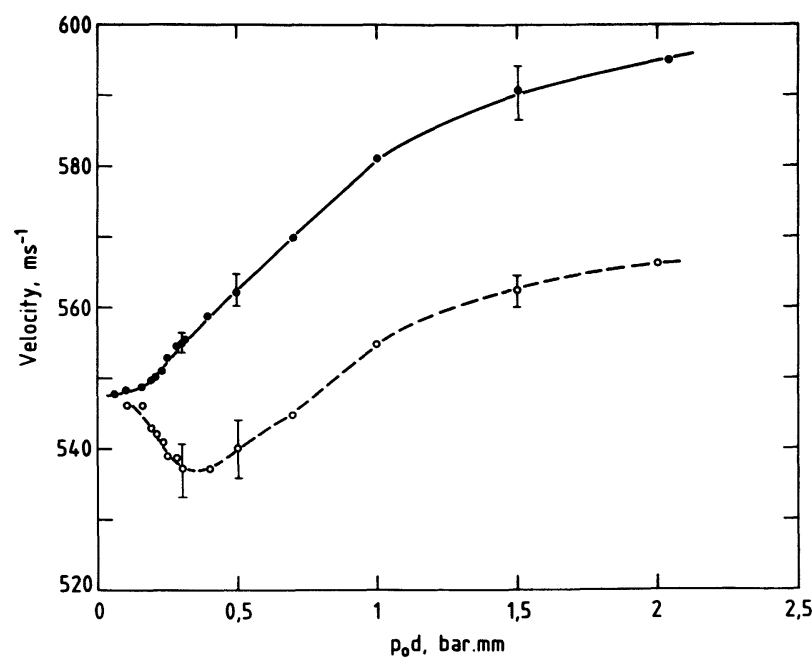

Fig. 5. - Velocities corresponding to the slow mode (dashed curve) and to the rapid mode (solid curve) of $\mathrm{Ar}^{+}$TOF's, as a function of the product $P_{0} \cdot d$. Typical experimental uncertainties are reported. release as kinetic energy of part of the energy of the cluster bonds.

It is worthwhile to observe that in figure 4 the TOF's corresponding to $\mathrm{Ar}^{+}$up to $(\mathrm{Ar})_{6}^{+}$have essentially the same shape. This suggests that these signals are all coming from the fragmentation of much larger clusters. These fragments being formed in the ionization chamber of the mass spectrometer, as was already mentioned.

\section{Experimental determination of the flux of free $\mathrm{Ar}$} atoms in the beam under various stagnation conditions.

The determination of the flux of free $\mathrm{Ar}$ atoms contained in the Ar beam can be done quantitatively by calculating the surface under the rapid mode in the $\mathrm{Ar}^{+} \mathrm{TOF}$.

In order to determine this surface, two cases were considered. First at low pressures when there is only one mode in the spectrum, the area of the peak in the time of flight has simply be measured. At higher pressures when two modes exist, a program for nonlinear least squares fit has been used [10]. In this

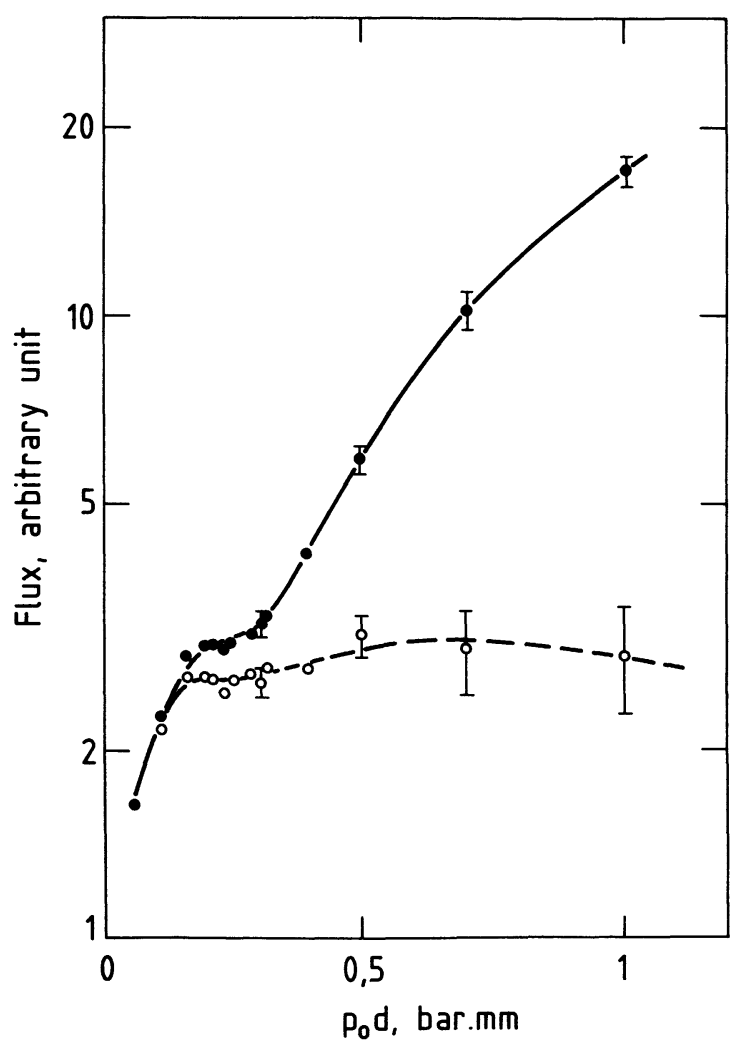

Fig. 6. - Flux of free atoms in a supersonic beam of Argon as a function of the product $P_{0} \cdot d$ (dashed curve). The total flux of Ar atoms, free or included in clusters, is given by the solid curve. Curves are normalized at the lower value of $P_{0}, d$. Typical experimental uncertainties are reported. 
case, the time of flight was fitted by the sum of two curves given by equation (1). The non-linear parameters were the two $V$ and the two $T$. By allowing these four parameters to vary or by fixing arbitrarily one or more of them, no significant difference was found in the resulting area of the fast mode corresponding to the monomers. Another method was also used : it consists in truncating the time fo flight by keeping only the part where we suspected that the only contribution was due to the monomers and fitting this part with the analytic form given by equation (1). Within 10 to $20 \%$ the two methods give the same results in what concerns the area of the fast mode.

The results are shown in figure 6 as a function of the product $P_{0} \cdot d$ over the range $0.15-1 \mathrm{bar} . \mathrm{mm}$. Of course, results for larger values of $P_{0}, d$ have not been reported since they correspond to situations where the rapid mode in the $\mathrm{Ar}^{+}$TOF is hardly visible. It turns out that the flux of free Ar atoms is about constant as the product $P_{0} \cdot d$ is increased. Correspondingly, the total flux of Ar atoms, free or included into clusters, as measured using the Pitot tube flux gauge show a marqued increase. The constancy of the free Ar atom flux can be interpreted by considering that it is determined essentially by the equilibrium vapor pressure of $\mathrm{Ar}$ under the conditions of final beam temperature, a quantity which do not depends that much on the value of $P_{0} \cdot d$. The monomer concentrations measured as a function of $P_{0} \cdot d$ agree with the results of J. Farges et al. obtained by an electron diffraction technique [11].

\section{Conclusion.}

Detailed TOF spectra have been measured in supersonic Ar beams using a mass spectrometer detector set to the mass of $\mathrm{Ar}^{+},(\mathrm{Ar})_{2}^{+}, \ldots$ up to $(\mathrm{Ar})_{6}^{+}$. Various stagnation conditions were used to generate the beam, thus allowing one to examine situations where very few clusters are present up to situations where the beam is mostly formed of clusters.

It turns out that when many clusters are present in the beam, TOF's measured for $\mathrm{Ar}^{+}$have a double mode structure. The rapid mode was attributed to the velocity distribution of $\mathrm{Ar}$ remaining as free atoms in the beam, and the slow mode to clusters that fragment to $\mathrm{Ar}^{+}$in the ionization chamber of the mass spectrometer. This effect has been used to quantitatively estimate the flux of free $\mathrm{Ar}$ atoms contained the beam. It appears that this flux is essentially constant as the stagnation conditions for generating the beam are changed over a wide range. In contrast, the total flux of Ar atoms, included or not in clusters increases a lot as the Ar backing pressure is increased.

\section{Acknowledgments.}

The authors wish to thank J. Berlande, J. M. Mestdagh and J. P. Visticot for fruitful discussion. They are grateful to C. Alcaraz, P. de Pujo and P. Meynadier for their help in the experimental work.

References

[1] Buck, U. and Meyer, H., J. Chem. Phys. 84 (1986) 4854.

[2] Farges, J., de Feraudy, M. F., Raoult, B.and TORChet, G., J. Chem. Phys. 78 (1983) 5067.

[3] Visticot, J. P., Mestdagh, J. M., Cuvellier, J. and Berlande, J., J. Chem. Phys. to appear February 1988.

[4] Hagena, O. F. and Obert, W., J. Chem. Phys. 56 (1972) 1793.

[5] FARgeS, J., J. Cryst. Growth 31 (1975) 79.

[6] Sherman, P. M., AIAA J. 9 (1971) 1628.

[7] Abuaf, N., Anderson, J. B., Anders, R. P., FenN, J. B. and MilleR, D. R., 5th symp. on Rarefied Gas Dynamics, p. 1317, Ed. C. L. Brundin (Academic Press) 1967.
[8] Cuvellier, J., Mestdagh, J. M., Berlande, J., De Pujo, P. and Binet, A., Revue Phys. Appl. 16 (1981) 679.

[9] Gallagher, R. J. and Fenn, F. B., J. Chem. Phys. 60 (1974) 3487.

[10] Osborne, M. R., Some aspects of non linear least squares calculation in Lootsme editor, « Numerical method for non linear optimization» (Academic Press, London) 1972.

[11] Farges, J., Feraudy, M. F., Raoult, B. and TORCHET, G., 10th International Symposium on Rarefied Gas Dynamics, J. Leith Potter Ed., Progress in Astronomics and Aeronautics, 51 (1977) 1117. 